\title{
MR Quantitative Susceptibility Imaging for the Evaluation of Iron Loading in the Brains of Patients with $\beta$-Thalassemia Major
}

\author{
D. Qiu, G.C.-F. Chan, J. Chu, Q. Chan, S.-Y. Ha, M.E. Moseley, and P.-L. Khong
}

\begin{abstract}
BACKGROUND AND PURPOSE: Patients with $\beta$-thalassemia require blood transfusion to prolong their survival, which could cause iron overload in multiple organs, including the heart, liver, and brain. In this study, we aimed to quantify iron loading in the brains of patients with $\beta$-thalassemia major through the use of MR quantitative susceptibility imaging.
\end{abstract}

MATERIALS AND METHODS: Thirty-one patients with thalassemia with a mean ( \pm standard deviation) age of 25.3 ( \pm 5.9 ) years and 33 age-matched healthy volunteers were recruited and underwent MR imaging at 3T. Quantitative susceptibility images were reconstructed from a 3D gradient-echo sequence. Susceptibility values were measured in the caudate nucleus, putamen, globus pallidus, red nucleus, substantia nigra, dentate nucleus, and choroid plexus. General linear model analyses were performed to compare susceptibility values of different ROIs between the patients with thalassemia and healthy volunteers.

RESULTS: Of the 31 patients, 27 (87.1\%) had abnormal iron deposition in one of the ROls examined. Significant positive age effect on susceptibility value was found in the putamen, dentate nucleus, substantia nigra, and red nucleus $(P=.002, P=.017, P=.044$, and $P=.014$, respectively) in the control subjects. Compared with healthy control subjects, patients with thalassemia showed significantly lower susceptibility value in the globus pallidus $(P<.001)$ and substantia nigra $(P=.003)$ and significantly higher susceptibility value in the red nucleus $(P=.021)$ and choroid plexus $(P<.001)$.

CONCLUSIONS: A wide range of abnormal susceptibility values, indicating iron overloading or low iron content, was found in patients with thalassemia. MR susceptibility imaging is a sensitive method for quantifying iron concentration in the brain and can be used as a potentially valuable tool for brain iron assessment.

ABBREVIATIONS: $\mathrm{QSM}=$ quantitative susceptibility mapping; $\mathrm{GP}=$ globus pallidus; $\mathrm{DN}=$ dentate nucleus; $\mathrm{SN}$ = substantia nigra; $\mathrm{CP}=\mathrm{choroid} \mathrm{plexus;} \mathrm{DFO}=$ deferoxamine; $\mathrm{L} \mathbf{l}=$ deferiprone

B eta-thalassemia major is a disease caused by genetic defects that lead to reduced production of hemoglobin. The patients require blood transfusion to prolong their survival, which could cause iron overload in multiple organs, including the heart, liver,

Received August 20, 2013; accepted after revision November 6.

From the Departments of Diagnostic Radiology (D.Q. P.-L.K.) and Pediatrics and Adolescent Medicine (G.C.-F.C., S.-Y.H.), The University of Hong Kong, Hong Kong, China; Department of Radiology and Imaging Sciences (D.Q.), Emory University, Atlanta, Georgia; Department of Radiology (J.C.), First Affiliated Hospital of Sun Yat Sen University, Guangzhou, China; Philips Healthcare Hong Kong (Q.C.), Hong Kong, China; and Department of Radiology (M.E.M.), Stanford University, Stanford, California.

Supported by the Children's Thalassemia Foundation, Hong Kong.

Please address correspondence to Deqiang Qiu, PhD, Emory University, Department of Radiology and Imaging Sciences, 1364 Rd, Clifton, CG06, Atlanta, GA 30322; e-mail: qiudeqiang@gmail.com or Pek-Lan Khong, MD, The University of Hong Kong, Department of Diagnostic Radiology, Room 406, Blk. K, Queen Mary Hospital, 102 Pokfulam Rd, Hong Kong; e-mail: plkhong@hkucc.hku.hk

Evidence-Based Medicine Level 2.

http://dx.doi.org/10.3174/ajnr.A3849 and brain. ${ }^{1}$ Iron chelation therapy is administrated to clear the excess iron. Whereas iron overload in the heart and liver may cause death, alterations of iron content in the brain may contribute to cognitive impairment, as observed in some patients with thalassemia, ${ }^{2,3}$ which has not been found to correlate with age and blood ferritin levels. Accurate assessment of iron content in the brains of patients with thalassemia could provide valuable information for individualized patient treatment. Conventionally, MR relaxometry on the basis of methods that use $\mathrm{T} 2$ and $\mathrm{T} 2{ }^{\star}$ mapping has been used to quantify iron in the brain. ${ }^{4}$ Recently, the application of measuring brain iron by use of phase value of MR imaging with $\mathrm{SWI}^{5,6}$ and quantitative susceptibility mapping $(\mathrm{QSM})^{7-12}$ has been evaluated for the quantification of iron content in patients with Parkinson disease. ${ }^{13}$ QSM is an MR imaging technique that measures the magnetic susceptibility of tissues, such as blood or iron content, through mathematically modeling their induced effects on the phase of signal. Although the derivation of a magnetic susceptibility image from the phase informa- 
tion obtained from MR imaging is an ill-posed problem, techniques have been established to overcome this by imposing smoothness or sparseness constraints on the susceptibility images. ${ }^{9-11}$ QSM has been successfully applied in multiple sclerosis, Parkinson disease, and so forth. A recent study ${ }^{14}$ has also established positive correlation between magnetic susceptibility values measured by use of QSM and iron content measured by $\mathrm{x}$-ray fluorescence imaging and inductively coupled plasma mass spectrometry. In the present study, we quantified the brain iron content in a cohort of patients with $\beta$-thalassemia major and compared this with healthy age-matched subjects. We hypothesize that there are significant differences in iron loading between patients with thalassemia and healthy control subjects as measured by QSM.

\section{MATERIALS AND METHODS Subjects}

Thirty-one (14 male) patients with $\beta$-thalassemia major were recruited for the study and 33 (17 male) age-matched healthy volunteers were recruited from the local community. The mean \pm standard deviation (SD) age of the patient group was $25.3 \pm 5.9$ years (range, 15.2-34.3 years); the mean \pm SD age of the control group was $26.1 \pm 4.1$ years (range 19.9-34.9 years). The study was approved by the institutional review board, and written informed consent was obtained from the subjects and/or their parent as appropriate. Among the 31 patients, 7 had hepatitis C, 16 had hypogonadism, and 7 had diabetes. The mean \pm SD number of years of transfusion for the patients was $24.6 \pm 5.9$ years, and average hemoglobin level of the patients before transfusion was $96.3 \pm 6.1 \mathrm{~g} / \mathrm{L}$.

\section{Chelation Therapy}

The patients received iron chelation therapy for an average of $20.9 \pm 5.2$ years. All 31 patients began their chelation therapy with deferoxamine (DFO). Of these 31 patients, 8 continued to receive DFO up to the dates of their MR imaging (DFO group), 6 changed treatment to deferiprone (L1; L1 group), 12 received additional treatment with L1, that is, combined DFO and L1 treatment ( $\mathrm{L} 1+\mathrm{DFO}$ group), and 5 changed treatment to deferasirox (Exjade group). The mean $\pm \mathrm{SD}$ treatment durations for the DFO, L1, L1+DFO, and Exjade groups with their current chelation agents at the time of MR imaging were 16.2 $\pm 8.2,4.6 \pm 2.3,2.8 \pm$ 1.3 , and $1.6 \pm 1.4$ years, respectively.

\section{Blood Ferritin Level}

Blood ferritin level was taken regularly every 2 months. The last reading before the MR imaging, the first reading after the MR imaging, and 1-year average were obtained for correlation with MR findings in patients with thalassemia. The mean \pm SD ferritin levels before and after MR imaging and the 1-year average were $4687.2 \pm 2715.8$ (range, $1119-111,062$ ) $\mu \mathrm{mol} / \mathrm{mL}, 4910.3 \pm$ 2853.9 (range, $1166-14,200$ ) $\mu \mathrm{mol} / \mathrm{mL}$, and $4868.5 \pm 2575.8$ (range, 1295-11,059) $\mu \mathrm{mol} / \mathrm{mL}$, respectively.

\section{Image Acquisition}

The MR imaging scan was performed with the use of an Achieva 3T scanner (Philips, Best, the Netherlands), and the protocol in- cluded a 3D gradient-echo sequence for susceptibility imaging $(\mathrm{FOV}=230 \mathrm{~mm}$, matrix $=256 \times 256$, section thickness $=2 \mathrm{~mm}$, $\mathrm{TR} / \mathrm{TE}=16 / 23 \mathrm{~ms}$ ). A T1-weighted image was acquired with the use of either MPRAGE $(\mathrm{FOV}=250 \mathrm{~mm}$, matrix $=252 \times 240$, section thickness $=1 \mathrm{~mm}$ ) or $2 \mathrm{D}$ inversion recovery turbo spinecho sequence $(\mathrm{IR}=800 \mathrm{~ms}, \mathrm{TR} / \mathrm{TE}=2000 / 20 \mathrm{~ms}, \mathrm{FOV}=230$ $\mathrm{mm}$, matrix $=296 \times 252$, section thickness $/$ gap $=4 / 1 \mathrm{~mm})$ for anatomic identification and image normalization.

\section{Image Processing}

Image processing was performed by use of Matlab (MathWorks, Natick, Massachusetts). The phase images were first unwrapped by use of PRELUDE (part of FSL [http://www.fmrib.ox.ac.uk/ fsl]) and the background field was removed by means of the spherical mean filtering technique. ${ }^{15}$ The background-removed phase image was then subject to an L1-norm constrained iterative reconstruction algorithm ${ }^{16}$ to calculate the magnetic susceptibility image from phase image. In the Fourier space, the relationship between magnetic field $f(\vec{k})$ (Fourier transformation of $f(\vec{r})$ ) and susceptibility distribution $\chi(\vec{k})$ (Fourier transformation of $\chi(\vec{r}))$ is point-wise multiplication with a kernel $C(\vec{k})$ :

$$
\begin{aligned}
f(\vec{k}) & =\chi(\vec{k}) \cdot C(\vec{k}) \\
C(\vec{k}) & =\left(\frac{1}{3}-\frac{k_{z}^{2}}{|\vec{k}|^{2}}\right),
\end{aligned}
$$

The relative magnetic field map $f(\vec{r})$ can be calculated from the processed phase map $\varphi(\vec{r})$ as:

$$
f(\vec{r})=-\frac{\varphi(\vec{r})}{\mathrm{TE} \cdot B_{0},}
$$

where TE is the echo time of the image and $B_{0}$ is the main magnetic field of the scanner. Reconstruction of magnetic susceptibility distribution $\chi(\vec{r})$ from the magnetic field map $f(\vec{r})$ is an illposed deconvolution problem because the convolution kernel $C(\vec{k})$ is zero at the cone surface $\left|\vec{k}^{2}\right|=3 \cdot k_{z}^{2}$. One approach to solving this problem is to condition the problem by imposing sparsity constraints on the susceptibility map and recast the problem as the following optimization problem:

4) $\chi(\vec{r})=\arg \min _{X}\left(|A \cdot X(\vec{r})-f(\vec{r})|_{2}+\rho \cdot|W \cdot X(\vec{r})|_{1}\right)$

where $A=i f f t \cdot C(\vec{k}) \cdot f f t$ is the forward transformation from susceptibility map to field map according to Equations 1 and 2, $f(\vec{r})$ is the relative field map calculated from the processed phase map according to Equation 3, $\mathrm{W}$ is a sparse transformation, that is, wavelet transformation, and $\rho$ is a tuneable parameter that controls the balance between the data consistency and solution sparsity in the transformed domain given by W. This algorithm is similar to the method previously proposed, except that wavelet transformation was used in this study instead of total variation as regularization term ${ }^{10}$ because wavelet transformation can provide a better sparsity of the images. ${ }^{17}$ An optimal $\rho$ was determined by a previously described method, ${ }^{18}$ in which the normalized mean square error of the reconstructed susceptibility map was plotted against different $\rho$ values, and the optimal $\rho$ was chosen to be a turning point of the curve.

We adopted a semi-automatic approach for the analysis of susceptibility value of multiple brain regions. With the use of 


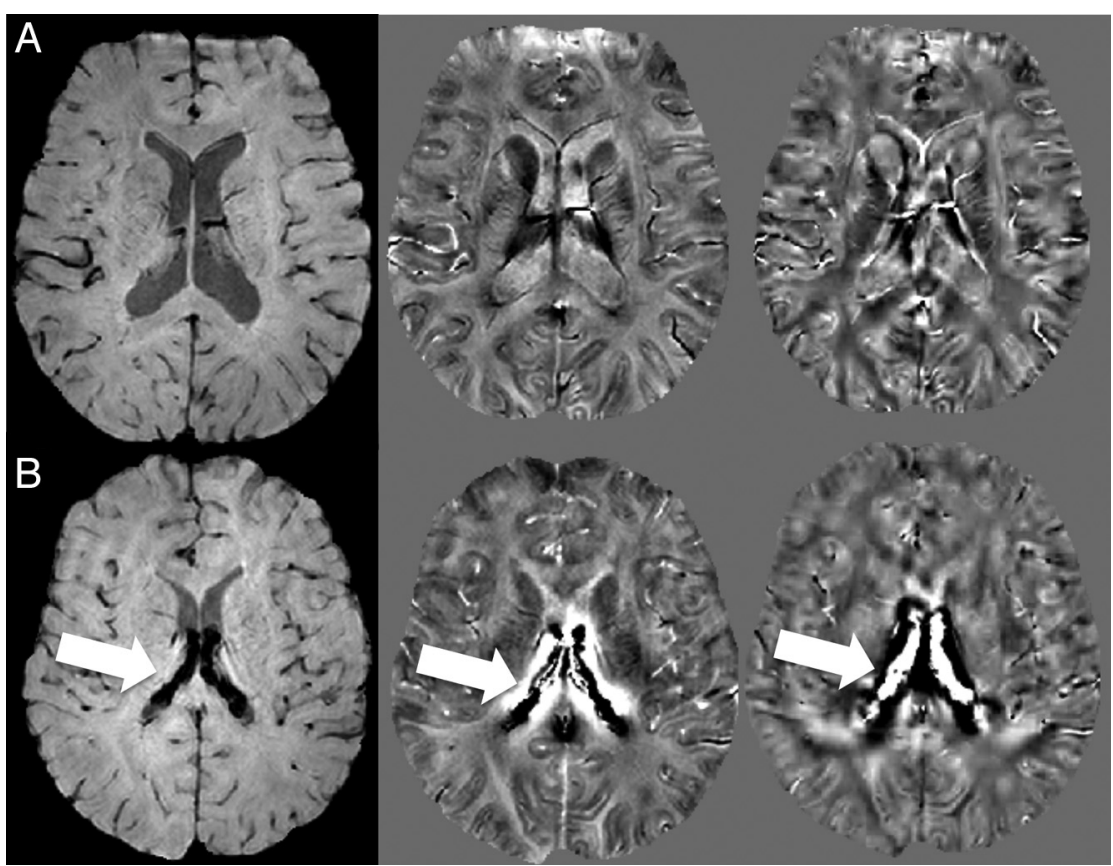

FIG 1. Left to right: Magnitude image, filtered phase image, and susceptibility image for (A) a healthy volunteer and (B) patient with thalassemia. For the patient with thalassemia, overloading of iron content in the choroid plexus can be observed as low signal intensity on the magnitude image and filtered phase image and high signal intensity in the susceptibility image (arrows).

pected normal susceptibility value $\left(\mathrm{SV}_{\mathrm{exp}}\right)$ for each ROI of each individual was calculated by use of $\mathrm{SV}=a+b \times$ age $+c \times$ sex, in which the coefficients $a, b$, and $c$ were determined from regression analysis from the healthy subjects. The age- and sex-corrected susceptibility value (cSV) was then calculated as $\mathrm{cSV}=\mathrm{SV}-\mathrm{SV}_{\text {exp }}$. The susceptibility value was defined to be low susceptibility value if $\mathrm{CSV}<-3^{\star} \mathrm{SD}$, in which $\mathrm{SD}$ indicates standard deviation of the residuals from the regression analysis from the healthy control subjects, and this suggests iron "underloading"; conversely, the susceptibility value was defined to be high susceptibility value if $\mathrm{cSV}>3{ }^{\star} \mathrm{SD}$, and this suggests iron overloading. On the basis of the cSV of the ROIs, the patients were then categorized as iron overloaded if at least 1 of the ROIs had high susceptibility value while no ROI had low susceptibility value; iron underloaded if at least 1 of the ROIs had low susceptibility value while none had high susceptibility value; "mixed"

SPM8 (Wellcome Department of Imaging Neuroscience, London, UK), the susceptibility image from every subject was normalized to Montreal Neurological Institute space by use of the respective magnitude image and the T1WI as medium. ROIs were then manually outlined on the group mean image of the normalized susceptibility maps and included bilateral caudate nucleus, putamen, globus pallidus (GP), red nucleus, substantia nigra $(\mathrm{SN})$, dentate nucleus (DN) and the choroid plexus (CP). The generated ROIs were then copied to each subject for measurement of susceptibility values and the mean susceptibility value was calculated as the mean value of all non-negative voxels within an ROI as defined above. We excluded voxels with negative susceptibility value to avoid the boundary effects and the inclusion of neighboring white matter voxels. The susceptibility values of left and right ROIs were then averaged for each anatomic region for further statistical analysis.

\section{Statistical Analysis}

Statistical analysis was performed with the use of SPSS (IBM, Armonk, New York). The Mann-Whitney $U$ test was performed to determine differences in age between patients and control subjects. A $\chi^{2}$ test was performed to determine difference in sex ratio between patients and control subjects. First, to evaluate the dependence of susceptibility values on age and sex among healthy control subjects, linear regression was performed by use of sex as a factor and age as a covariate. Second, general linear model analysis was performed to determine susceptibility value difference for different ROIs between patients and control subjects, controlling for the effect of age.

To further characterize the iron loading status of each patient, each ROI from each patient was classified according to the regression analysis result from the healthy control subjects. The ex- findings if low susceptibility value was found in 1 ROI and high susceptibility value was found in another ROI; and normal if no ROI had low susceptibility value or high susceptibility value.

Pearson correlation was performed between susceptibility values of each ROI with blood ferritin levels taken within 2 months before and after the MR image as well as its average over 1 year. General linear model analysis was performed to compare susceptibility value of each ROI between patients receiving different types of chelation therapy after controlling for the effect of age. A value of $P<.05$ was considered statistically significant.

\section{RESULTS}

\section{Qualitative Observations}

Qualitatively, QSM was able to show regions of high iron deposition at the expected locations in the brain. Subcortical structures including putamen, GP, SN, and red nucleus can be nicely depicted in QSM images (Fig 1A). In some patients, a high amount of iron deposition was shown as high signal intensity in susceptibility imaging in regions including the $\mathrm{CP}$ (Fig $1 B$ ). Figure 2 shows the placement of ROIs on the mean susceptibility image.

\section{Relationship between Susceptibility Value and Age and Sex among Control Subjects}

Table 1 shows the result of linear regression of the susceptibility value on the control group with age and sex as independent variables. A significant positive effect of age on susceptibility value was found in the putamen, DN, SN, and red nucleus, suggesting higher level of iron concentration with increasing age in these regions ( $P=.002, .017, .044$, and .014 , respectively). In the DN, significantly higher susceptibility value was found in the women as compared with men after controlling for the effect of age ( $P=$ .018). In other regions except for the $\mathrm{SN}$, the susceptibility value 


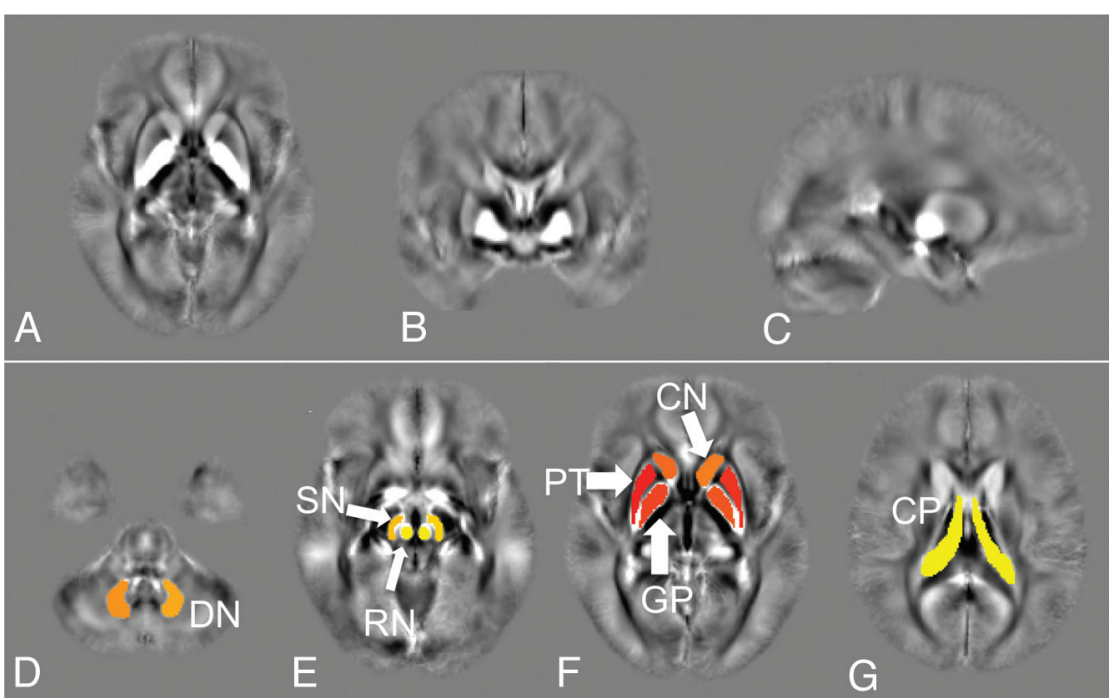

FIG 2. Mean susceptibility map on (A) axial, $(B)$ coronal, and (C) sagittal plane and (D-G) placement of ROls on the mean susceptibility map, including the caudate nucleus (CN), putamen (PT), globus pallidus (GP), red nucleus (RN), substantia nigra (SN), dentate nucleus (DN) and choroid plexus (CP).

Table 1: Results of linear regression of the susceptibility value on the control group with age and sex as independent variables

\begin{tabular}{lccccc}
\hline ROI & $\begin{array}{c}\text { Intercept } / 10^{-2} \\
\text { ppm }\end{array}$ & $\begin{array}{c}\text { Age } / 10^{-3} \\
\text { ppm }\end{array}$ & $\begin{array}{c}\text { Sex } / 10^{-3} \\
\text { ppm }\end{array}$ & $\begin{array}{c}P \text { Value } \\
\text { of Age }\end{array}$ & $\begin{array}{c}P \text { Value } \\
\text { of Sex }\end{array}$ \\
\hline PT & 0.45 & 0.51 & 1.15 & .002 & .346 \\
GP & 7.22 & 0.18 & 8.26 & .729 & .057 \\
CN & 1.55 & 0.18 & 0.30 & .338 & .837 \\
DN & 1.60 & 1.10 & 8.68 & .017 & .018 \\
SN & 4.35 & 1.22 & -3.49 & .044 & .455 \\
RN & 1.26 & 1.72 & 1.07 & .014 & .841 \\
CP & 3.83 & -0.341 & 9.75 & .763 & .284 \\
\hline
\end{tabular}

Note:-Significant positive age effect on susceptibility value was found in PT, DN, SN, and RN, suggesting higher level of iron concentration with increasing age. In the DN, significantly higher susceptibility value was found in women compared with men after controlling for the effect of age. In other regions except for the $\mathrm{SN}$, the susceptibility value was also higher in women, though these differences were not statistically significant. These findings suggest that in the majority of brain regions, iron concentration increases with age and is higher among women.

SD indicates standard deviation; PT, putamen; GP, globus pallidus; CN, caudate nucleus; DN, dentate nucleus; SN, substantia nigra; $\mathrm{RN}$, red nucleus; $\mathrm{CP}$, choroid plexus.

was also higher in women, though these differences were not statistically significant. These findings suggest that in most of the brain regions, iron concentration increases with age and is higher among women.

\section{Iron Deposition in Patients with Thalassemia}

Table 2 shows mean and SDs of susceptibility values of different ROIs between healthy control subjects and patients with thalassemia, as well as the $P$ value for group effect by use of the general linear model after controlling for the effect of age. Significantly lower susceptibility value was found in patients with thalassemia compared with healthy control subjects in the GP $(P<.001)$ and the SN $(P=.003)$; significantly higher susceptibility value was found in patients with thalassemia in the red nucleus $(P=.021)$ and the CP $(P<.001)$.

\section{Classification of Patients}

On the basis of the results from the linear regression analysis on healthy control subjects, we established a normal range of the susceptibility value by use of 3 times the SD of the residues as cutoff points. Out of the 31 patients, 27 (87.1\%) had abnormal iron deposition in at least 1 of the ROIs. Six had low susceptibility value in at least 1 of the ROIs and normal susceptibility value in other ROIs. Thirteen of the patients had high susceptibility values in at least 1 of the ROIs and normal susceptibility value in other ROIs, 12 of which involved the CP. Eight of the patients had mixed abnormal susceptibility value, with at least 1 of the ROIs having higher susceptibility value and at least 1 other ROI having lower susceptibility values.

Table 3 shows the number of patients with thalassemia with iron overloading or underloading in each ROI. The largest number of patients was found to have abnormal susceptibility value in the CP $(n=20)$, followed by the GP $(n=12)$. As a control analysis, the same analysis was performed on healthy volunteers, and the results showed that all ROIs had normal susceptibility value.

\section{Correlation between Blood Ferritin Level and Susceptibility Value}

There was no significant correlation between measures of ferritin level and susceptibility value of the ROIs.

\section{Susceptibility Value and Chelation Agents}

Table 4 shows means and SDs of susceptibility values in the brain regions among patients receiving different chelation agents. No significant differences between patients receiving different chelation agents were found.

\section{DISCUSSION}

In this study, we applied QSM in studying brain iron loading in a group of patients with thalassemia and age-matched control subjects. The prevalence of "abnormal" iron concentration was $87.1 \%$ in our patient cohort, and we found large variations of iron concentration among the patients. Approximately two-thirds of the patients had high susceptibility value in the CP (20/31), suggesting overloaded iron content in this region, whereas reduced or "underloaded" iron concentration was found in the GP in approximately one-third of the patients (11/31).

Among the control subjects, higher susceptibility value was found with increasing age in multiple brain regions, which is consistent with the literature. ${ }^{19}$ The landmark work of Hallgren and Sourander, ${ }^{20}$ whose data still provide the largest quantitative survey of brain iron content with age, indicates that iron typically increases rapidly from birth until approximately 20 years of age 
Table 2: Mean (standard deviation) of susceptibility values of different ROls between healthy control subjects and patients with thalassemia and $\boldsymbol{P}$ values for group effect with the use of the general linear model after controlling for effects of age and sex

\begin{tabular}{|c|c|c|c|c|c|c|c|}
\hline & PT & GP & $\mathrm{CN}$ & DN & SN & RN & $\mathrm{CP}$ \\
\hline Control subjects $\left(10^{-2} \mathrm{ppm}\right)$ & $1.82(0.40)$ & $8.23(1.24)$ & $2.04(0.41)$ & $4.94(1.19)$ & $7.42(1.35)$ & $5.75(1.63)$ & $3.00(0.63)$ \\
\hline Patients ( $\left.10^{-2} \mathrm{ppm}\right)$ & $1.91(0.63)$ & $6.58(2.23)$ & $2.04(0.62)$ & $4.55(1.81)$ & $6.33(1.37)$ & $6.67(2.52)$ & $7.17(4.55)$ \\
\hline$P$ value & .248 & $<.001$ & .842 & .246 & .003 & .021 & $<.001$ \\
\hline
\end{tabular}

Note:-Significantly lower susceptibility value was found in patients with thalassemia compared with healthy control subjects in GP and SN; significantly higher susceptibility value was found in patients with thalassemia in $\mathrm{RN}$ and $\mathrm{CP}$.

PT indicates putamen; GP, globus pallidus; CN, caudate nucleus; DN, dentate nucleus; SN, substantia nigra; RN, red nucleus; CP, choroid plexus.

Table 3: Number of patients with thalassemia with iron overloading or underloading in each ROI

\begin{tabular}{lccccccr}
\hline & PT & GP & CN & DN & SN & RN & CP \\
\hline Iron overload & 1 & 0 & 2 & 1 & 0 & 3 & 20 \\
Iron underload & 0 & 11 & 0 & 2 & 1 & 0 & 0 \\
\hline
\end{tabular}

Note:-PT indicates putamen; GP, globus pallidus; $C N$, caudate nucleus; $D N$, dentate nucleus; $S N$, substantia nigra; RN, red nucleus; $\mathrm{CP}$, choroid plexus.

Table 4: Susceptibility values among patients receiving different chelation agents

\begin{tabular}{lccccc}
\hline & \multicolumn{4}{c}{ Mean (SD) $/ 10^{-2}$ Ppm } & \\
\cline { 2 - 4 } & DFO & L1 & DFO+L1 & Exjade & P Value \\
\hline PT & $1.82(0.69)$ & $2.09(0.50)$ & $1.81(0.59)$ & $2.07(0.85)$ & .201 \\
GP & $7.64(2.07)$ & $6.69(3.50)$ & $5.62(1.73)$ & $7.06(1.06)$ & .296 \\
CN & $2.37(0.60)$ & $1.66(0.49)$ & $1.98(0.54)$ & $2.09(0.80)$ & .232 \\
DN & $4.95(1.53)$ & $3.50(1.57)$ & $4.44(2.08)$ & $5.44(1.55)$ & .064 \\
SN & $6.87(1.77)$ & $5.89(1.61)$ & $6.28(1.15)$ & $6.08(0.86)$ & .573 \\
RN & $6.01(3.23)$ & $7.26(1.85)$ & $6.86(1.90)$ & $6.55(3.68)$ & .801 \\
CP & $7.38(4.28)$ & $7.03(3.63)$ & $7.47(6.09)$ & $6.24(1.75)$ & .955 \\
\hline
\end{tabular}

Note:-No significant difference was found in susceptibility values between patients receiving different chelation agents.

DFO indicates deferoxamine; L1, deferiprone; SD, standard deviation; PT, putamen; CN, caudate nucleus; RN, red nucleus.

for nearly all brain regions. After this age, brain iron increases less rapidly and in some regions approaches a distinct plateau in middle age.

Iron overload in $\beta$-thalassemia occurs through multiple pathways, including extensive destruction of senescent native and transfused red blood cells, increased intestinal iron absorption caused by tissue hypoxia, apoptosis of defective erythroid precursors, and peripheral hemolysis. ${ }^{21}$ Whether abnormal systemic iron level directly leads to changes in the brain iron level is unclear. It is expected that the brain's iron levels would be well regulated and maintained according to its own mechanism and metabolic needs, and the existence of the blood-brain barrier would further reduce the influence of blood iron levels on brain iron level. Nonetheless, it is conceivable that blood iron levels may have some degree of modulation effect on the brain iron levels. It is interesting to note that mixed levels of abnormal brain iron content were observed among the patients with thalassemia in the present study, with some regions having excessive iron concentration such as the $\mathrm{CP}$ and less so, the red nucleus, and some regions having low iron concentration such as the GP and less so, the SN and DN. Also, the dynamic interactions between the effects of regular blood transfusion and iron chelation therapy may contribute to the regional variations in brain iron level among the different regions. It has been shown that the $\mathrm{CP}$, which forms the blood-CSF barrier, is an important interface for brain iron homeostasis and has abundant proteins related to iron transportation, ${ }^{22}$ which may contribute to the excessive iron content in this region among patients with thalassemia. It is noteworthy that iron content in brain regions increases with age among healthy subjects. ${ }^{23}$ Because of disruption of sex hormone production, ${ }^{24}$ patients with $\beta$-thalassemia have delayed maturation, which may contribute to the observed lower iron content in the GP and the DN among patients with $\beta$-thalassemia. Moreover, patients with $\beta$-thalassemia are prone to chronic systemic illnesses, including hypogonadism, hepatitis C, diabetes, and so forth. All these may be contributory to the effects on the brain and cognition. Thus, the exact physiologic mechanism underlying the abnormal iron distribution in patients with thalassemia is complex and remains to be fully elucidated.

Although the effects of iron overload on heart and liver dysfunction have been extensively studied because of their early impact on survival, the effect of alteration of iron content in the central nervous system remains unclear and the literature to date is limited. It has been found that patients with thalassemia have neuropsychological impairment in the domains of abstract reasoning, attention, and memory. ${ }^{2}$ Hemosiderosis, toxicity from chelating agents, and chronic hypoxia have been postulated to be the contributory factors of these cognitive dysfunctions. In our current study, we found that most patients had either higher or lower levels of iron in at least 1 region evaluated, but with variations across regions and subjects. Previous study with the use of T2 mapping showed higher brain iron loading in patients with $\beta$-thalassemia. ${ }^{25}$ Although the exact cause of the abnormal brain iron level is still unclear, the presence of these brain iron level abnormalities as shown in the current study may have implications in cognitive functioning. High brain iron level is associated with high oxidative stress, and this has been implicated in many neurologic diseases including Parkinson disease, Alzheimer disease, multiple sclerosis, and so forth. ${ }^{4}$ On the other hand, low iron content has been shown to adversely affect the dopaminergic-opiate system and the cholinergic system ${ }^{26}$ and thus may also affect cognition. In addition, factors associated with chronic illness leading to regular school absence, physical and social restrictions, abnormal mental state, rather than the disease per se, could also play a potential role in the development of cognitive dysfunction in patients with thalassemia., ${ }^{2,3}$

It would have been interesting to evaluate the effect of the various chelation agents on iron deposition. Although our preliminary results showed no significant differences, this retrospective study was limited by the confounding variable of different treatment times and the small sample size. However, it is interest- 
ing to note that patients treated with L1 had the lowest iron loads in the DN, and the ANOVA analysis approached statistical significance. L1, which crosses the blood-brain barrier, has been found useful in lowering iron levels in the DN of patients with Friedreich ataxia. $^{27}$

Other limitations of the current study are that we did not acquire quantitative liver or cardiac iron measures or neuropsychological assessments. Future controlled studies with a larger cohort including these measures are warranted. Finally, although susceptibility values obtained in our study are generally lower than what was reported previously, ${ }^{28}$ this is possibly caused by the combination of differences in subject cohort, ROI placements, and the selection of regularization parameters in the dipole deconvolution. However, because the parameters used were the same between patients and control subjects, the susceptibility values obtained are directly comparable.

\section{Conclusions}

We have shown that quantitative susceptibility mapping is sensitive to iron deposition in the brain. The nature of abnormal iron content varies across different anatomic regions in patients with thalassemia. QSM is potentially a valuable tool for iron assessment, both on an individual basis for which treatment may be tailored and for use in clinical trials.

Disclosures: Deqiang Qiu—RELATED: Grant: Children's Thalassemia Foundation, Hong Kong.* Godfrey Chi-Fung Chan-UNRELATED: Grants/Grants Pending: Children's Thalassemia Foundation*; Payment for Lectures (including service on speakers bureaus): Lectures to colleagues in China and Macao, ${ }^{\star}$ Comments: Paid by Macao Central Hospital (a government hospital) for contribution as teaching consultant; Patents (planned, pending or issued): University of Hong Kong (Versitech), , Comments: Collagen microsphere for stem cells. Queenie Chan-UNRELATED: Employment: Philips Electronics (Hong Kong). Pek-Lan Khong-RELATED: Grant: Children's Thalassemia Foundation*; UNRELATED: Grants/Grants Pending: SK Yee Medical Foundation Grant for the Sick and Poor; Travel/Accommodations/Meeting Expenses Unrelated to Activities Listed: University of Hong Kong ( ${ }^{*}$ money paid to institution).

\section{REFERENCES}

1. Olivieri NF, Brittenham GM. Iron-chelating therapy and the treatment of thalassemia. Blood 1997;89:739-61

2. Economou M, Zafeiriou DI, Kontopoulos E, et al. Neurophysiologic and intellectual evaluation of beta-thalassemia patients. Brain Dev 2006;28:14-18

3. Monastero R, Monastero G, Ciaccio C, et al. Cognitive deficits in beta-thalassemia major. Acta Neurol Scand 2000;102:162-68

4. Brass SD, Chen NK, Mulkern RV, et al. Magnetic resonance imaging of iron deposition in neurological disorders. Topics Magn Reson Imaging 2006;17:31-40

5. Haacke EM, Mittal S, Wu Z, et al. Susceptibility-weighted imaging: technical aspects and clinical applications, part 1. Am J Neuroradiol 2009;30:19-30

6. Mittal S, Wu Z, Neelavalli J, et al. Susceptibility-weighted imaging: technical aspects and clinical applications, part 2. AJNR Am J Neuroradiol 2009;30:232-52

7. de Rochefort L, Brown R, Prince MR, et al. Quantitative MR susceptibility mapping using piece-wise constant regularized inversion of the magnetic field. Magn Reson Med 2008;60:1003-09

8. Kressler B, de Rochefort L, Liu T, et al. Nonlinear regularization for per voxel estimation of magnetic susceptibility distributions from MRI field maps. IEEE Trans Med Imaging 2010;29:273-81

9. Li W, Wu B, Liu C. Quantitative susceptibility mapping of human brain reflects spatial variation in tissue composition. NeuroImage 2011;55:1645-56

10. Liu T, Liu J, de Rochefort L, et al. Morphology enabled dipole inversion (MEDI) from a single-angle acquisition: comparison with COSMOS in human brain imaging. Magn Reson Med 2011;66: 777-83

11. Shmueli K, de Zwart JA, van Gelderen P, et al. Magnetic susceptibility mapping of brain tissue in vivo using MRI phase data. Magn Reson Med 2009;62:1510-22

12. Wharton S, Bowtell R. Whole-brain susceptibility mapping at high field: a comparison of multiple- and single-orientation methods. NeuroImage 2010;53:515-25

13. Lotfipour AK, Wharton S, Schwarz ST, et al. High resolution magnetic susceptibility mapping of the substantia nigra in Parkinson's disease. J Magn Res Imaging 2012;35:48-55

14. Zheng W, Nichol H, Liu S, et al. Measuring iron in the brain using quantitative susceptibility mapping and $\mathrm{X}$-ray fluorescence imaging. NeuroImage 2013;78:68-74

15. Schweser F, Deistung A, Lehr BW, et al. Quantitative imaging of intrinsic magnetic tissue properties using MRI signal phase: an approach to in vivo brain iron metabolism? NeuroImage 2011;54: 2789-807

16. Qiu D, Zaharchuk G, Feng S, et al. Quantitative Susceptibility Imaging using $\mathrm{L} 1$ regularized reConstruction with sparsity promoting transformation: SILC. Proceedings of International Society of Magnetic Resonance in Medicine Annual Meeting 2011;19:4470

17. Chui CK. An Introduction to Wavelets. San Diego: Academic Press; 1992

18. Wu B, Li W, Guidon A, et al. Whole brain susceptibility mapping using compressed sensing. Magn Reson Med 2012;67:137-47

19. Pfefferbaum A, Adalsteinsson E, Rohlfing T, et al. MRI estimates of brain iron concentration in normal aging: comparison of field-dependent (FDRI) and phase (SWI) methods. NeuroImage 2009;47: 493-500

20. Hallgren B, Sourander P. The effect of age on the non-haemin iron in the human brain. J Neurochem 1958;3:41-51

21. Rund D, Rachmilewitz E. Beta-thalassemia. $N$ Engl J Med 2005;353:1135-46

22. Rouault TA, Zhang DL, Jeong SY. Brain iron homeostasis, the choroid plexus, and localization of iron transport proteins. Metab Brain Dis 2009;24:673-84

23. Li W, Wu B, Batrachenko A, et al. Differential developmental trajectories of magnetic susceptibility in human brain gray and white matter over the lifespan. Hum Brain Mapp 2014;35:2698-713

24. Moshtaghi-Kashanian GR, Razavi F. Ghrelin and leptin levels in relation to puberty and reproductive function in patients with betathalassemia. Hormones 2009;8:207-13

25. Metafratzi Z, Argyropoulou MI, Kiortsis DN, et al. T(2) relaxation rate of basal ganglia and cortex in patients with beta-thalassaemia major. Br J Radiol 2001;74:407-10

26. Youdim MB. Brain iron deficiency and excess; cognitive impairment and neurodegeneration with involvement of striatum and hippocampus. Neurotox Res 2008;14:45-56

27. Pandolfo M, Hausmann L. Deferiprone for the treatment of Friedreich's ataxia. J Neurochem 2013;126(Suppl 1):142-46

28. Lim IA, Faria AV, Li X, et al. Human brain atlas for automated region of interest selection in quantitative susceptibility mapping: application to determine iron content in deep gray matter structures. NeuroImage 2013;82:449-69 